\title{
Intercultural Negotiation with Virtual Humans: The Effect of Social Goals on Gameplay and Learning
}

\author{
Amy Ogan ${ }^{1}$, Vincent Aleven ${ }^{1}$, Julia Kim², Christopher Jones ${ }^{1}$
}

\author{
${ }^{1}$ Carnegie Mellon University, \\ Pittsburgh, Pennsylvania, USA \\ $\{$ aeo, aleven, jones $\} @$ andrew.cmu.edu
}

\begin{abstract}
One innovative use of digital games is to facilitate learning skills with social components by simulating human behavior with virtual humans. We investigate learners' social goals to understand how they help learners learn intercultural skills from virtual humans in BiLAT, a virtual world that teaches cross-cultural negotiation. We hypothesize that students learn more when they approach the simulation as a social interaction rather than taking a trial-anderror approach perhaps characteristic of video gaming. In a randomized controlled experiment with 59 participants, we found that participants improved cross-cultural negotiation skills through game play. Our hypothesis that participants given an explicit social goal would learn more than those given task-related goals was not confirmed. We did, however, find a positive relation between students' self-reported social goals, regardless of condition, and their learning results. This relation was confirmed through analysis of $\log$ data. Although it is still an open question how best to promote students' approaching a simulation with a social orientation, the results underline the importance of such goals.
\end{abstract}

Keywords: social motivation, virtual environments, intelligent agents

\section{Introduction}

Virtual humans are increasingly being used as pedagogical agents [2], and one of their most intriguing roles may be in facilitating the learning of social or interpersonal skills, such as conflict resolution, in game-based instructional systems [14]. These skills are traditionally taught in environments like business training or foreign language classes, using methods like role-playing, or lecture and discussion. Unfortunately, these methods are either highly resource-intensive or lack an experiential component where learners practice these skills [10]. Computer-based simulations, which are growing more realistic, offer a major advantage for social learning by providing a cheaper solution to a much larger number of learners. Social learning systems have leveraged video game and learning technologies to create immersive environments with virtual human characters that simulate interpersonal interactions with complex behavioral models (see [11] for a review of intercultural systems). Recent advances in artificial intelligence and cognitive modeling now permit rich modeling of affect, culture, and more in virtual humans [e.g., 18]. The virtual humans' models drive the interaction that theoretically leads to learning. BiLAT, the game-based system in which we situate this work, is a virtual 
environment that supports learners in developing cross-cultural skills in the context of a negotiation task [8]. In this game, virtual humans take on a role that would typically be played by a human from another culture, in which they gesture, speak, and behave in ways that would be appropriate in that culture. Such games allow us to study the effects of virtual humans on social learning.

Unlike many pedagogical agents in the role of teacher, these virtual humans do not provide support for problem solving, but rather learners benefit from the practice they receive through interacting with them, as they would from role-playing practice with a human. If we are to make optimal use of virtual humans as agents for social learning, it is helpful to understand our interactions with them. Related work supports the hypothesis that humans interact socially with computers. According to a wide-ranging series of studies by Reeves and Nass [15], people have an inherent bias that leads them to react to media like computers in fundamentally social ways. For example, people rate more highly computers that are endowed with a similar "personality" to their own. Recently, others have shown similar results investigating virtual humans, who can evoke responses that follow social psychology theory in automated processes such as proxemics (differences in physical distances between people) [1].

However, there are contradictory findings when more conscious, cognitive functions are involved [16]. Bailenson, Blascovich, Beall, \& Loomis propose that social responses to virtual humans are forthcoming in low-level responses such as proxemics, while high-level responses such as having a meaningful conversation are governed by the human participants' belief in the agency of the virtual human [1]. Learning may very well be one such high-level area that requires attention and processing [3]. Okita, Bailenson, and Schwartz [12] found that learners who believed there was a human behind an avatar in a virtual environment exhibited better learning, more attention, and higher arousal than learners who believed they were interacting with a machine. One explanation for this result is that learners believe they are taking a socially relevant action when they interact with a human, and thus pay more attention and feel more accountable. It is clear that our social responses to technology are not the same in all cases. If learners are to benefit from these interactions it may be necessary for them to perceive the agents as social beings.

Even if learners do not produce social responses naturally, it may be possible to introduce social orientations into human-agent interactions. A significant area of research involves learners' motivational orientations towards learning environments [5]. In a domain like culture that focuses on social interactions, social motivation can have a great influence on learning [20]. Motivation is a goal-directed process that instigates or sustains behavior [17]. When cultural differences arise, people with different cultural identities are often categorized as members of an "outgroup" [13], which can exacerbate biases and lead to social goals like the desire to be seen as superior to the outgroup [19]. These motives may be detrimental to learning about a new culture. On the other hand, if the learner possesses social goals such as a need for affiliation or the desire to conform to social rules, learning may increase. It is an open question whether such goals can be explicitly manipulated in a way that improves learning in environments like BiLAT, in which learners interact with virtual characters from a different culture.

Social goals have been studied in human-human contexts outside of learning, in a way that might lead to successful interventions for learning purposes. Negotiation 
researchers have shown that having a sense of a shared group identity, as indicated by holding social goals such as the desire for affiliation, can lead towards a win-win perspective and even increase negotiation outcomes for both parties [e.g., 21]. Social goals have also been manipulated to reduce outgroup bias in cross-cultural contact with successful results [6]. To study goal orientations, researchers give brief instructions that encourage specific goals for the task, which has been shown to have a significant impact on students' goals [5]. In the same way, we can give learners social goals to influence their orientation towards virtual humans.

In this paper we describe an empirical study that investigates the effects of manipulating learners' social goals on their learning and gameplay. This research explores whether students learn intercultural competence skills from BiLAT, whether learners with a social orientation towards virtual humans show increased learning, and whether giving learners social goals for interacting with virtual humans increases their social orientation. This paper presents a randomized controlled study in which learners played the game with or without the addition of an explicit social goal introduced into the interface of BiLAT. We present an investigation of participants' goals while using the system, their learning results, and the differences in participants' gameplay across groups.

\section{Game Context}

The environment we use for our investigation is BiLAT [8], a game-based simulation for practicing bilateral meetings in a cross-cultural context. BiLAT integrates research technologies such as virtual human characters and intelligent tutoring support. Learners enter the practice environment where they interact with virtual characters. Scenarios derived from real-world events drive the game experience. The learner is put into the role of a U.S. Army officer tasked with meeting with Iraqi townspeople to accomplish peace-keeping and rebuilding missions. The learner is given concrete, negotiation task-related goals for each meeting.

BiLAT was designed to address learning objectives related to negotiation generally as well as the specific cultural knowledge and skills that support more effective negotiations in the Iraqi culture. A primary learning objective is considering the meeting counterpart's interests in order to achieve "win-win" results. To play, the learner begins by preparing for a meeting in the "prep room." Here, the learner learns about the character from a variety of sources of varying degrees of trustworthiness, similar to gathering such information in a real world situation. The learner then moves into the meeting (see Fig 1). The learner communicates with each BiLAT character by selecting from a menu of hand-authored communicative actions. Underlying each virtual character is a social simulation with a model of culture and personality that guides his or her responses. The character responds to players' actions in both text and synthesized speech as well as culturally-appropriate non-verbal behaviors. Once the meeting objectives have been obtained, the meeting is complete and the player will receive the next set of objectives for the scenario and new characters who might help them achieve those objectives. 


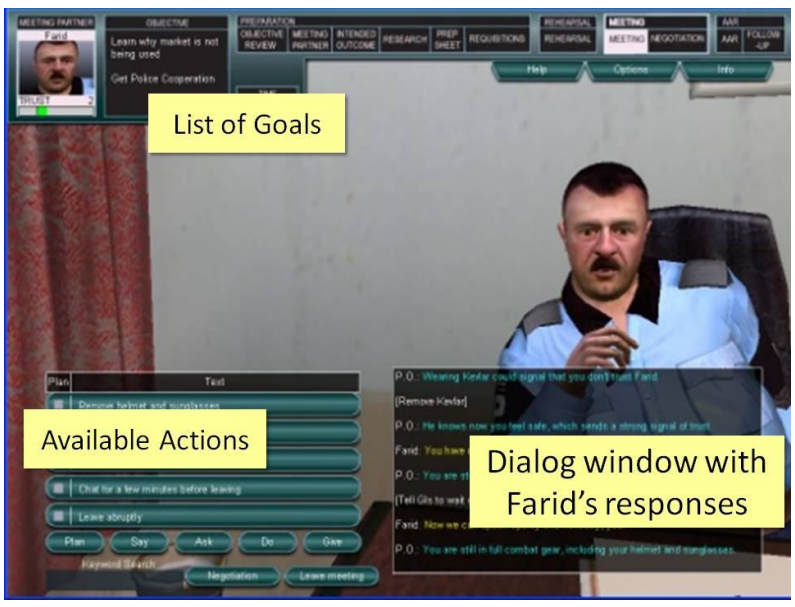

Fig 1. A meeting in BiLAT with police captain Farid, with the goal of solving a problem with a market in an Iraqi town

\section{Experimental Study}

In the BiLAT environment, we ran a between-subjects randomized controlled study to investigate student interactions and learning results with different goals. Participants were randomly assigned to either the task condition in which they viewed only taskrelated goals, or the social goal group with an additional social goal. We incorporated this goal as a single sentence which had to be selected each time participants started a meeting, and which was also available in the list of goals throughout the meeting (see Fig 1). This social goal was chosen by reflecting on the main focus of both the negotiation and cultural learning objectives, which has been found to improve learning: perspective taking. Accordingly, the social goal learners received was, "Come to understand Farid's point of view," where Farid was replaced with the name of the current meeting partner. Our hypotheses for this study were:

H1: Participants would show learning from the system.

$H 2$ : The social goal condition would have more social goals for interaction with the virtual characters.

H3: Students with social goals would perform better on overall learning measures.

H4: Students with social goals would have more social patterns of interaction with the virtual characters.

Participants were 59 learners (32 males, 27 females, mean age $=20.8, S D=2.71$ ) from two universities. In a demographics questionnaire we asked about participants' formal negotiation training $(M=1.20, S D=0.58)$, and knowledge of Arab cultures $(M=1.98, S D=1.22)$, on a scale of 1 (none) to 7 (extensive). 


\subsection{Measures}

Assessment of intercultural competence is not a trivial task. It is an ill-defined domain; there is not always a clear distinction between right and wrong answers, and even experts at times may disagree. We developed an assessment to test a learner's ability to develop an accurate model of the virtual characters and the tasks in the scenario. Specifically, we asked participants to rate the truth of various items relating to the task and to the character (e.g., "Farid could be described as a family-oriented man"). These items were taken from the information participants received in the "prep room," information which they knew might be accurate or misleading. Participants evaluated the items as true, false, or unknown. Throughout the course of a meeting with a character, successful participants should be able to revise their understanding of the character and the task through their interactions, and make more appropriate judgments about the accuracy of the information from the sources in the prep room. We called this measure Information Integration.

For our second measure we introduced a selection of questions from a validated instrument called the Cultural Assimilator [4]. In this assessment, participants read a scenario about people experiencing a foreign culture and chose the best of four possible cultural explanations for the events that occurred. The Cultural Assimilator deals with scenarios from both Iraqi and other cultures therefore testing whether students can transfer their knowledge to novel situations. Each version of the assessment contained six items, and in the study pretests and posttests were counterbalanced within conditions with isomorphic items.

Finally, we wanted to determine whether our manipulation had the desired effect on participants' goals in the game. We therefore asked them to list their goals in free text after meeting with a character.

\subsection{Procedure}

Participants began by taking a demographics questionnaire and then watching a video about the concepts and skills related to the learning objectives. This video gave participants an introduction to the material they then practiced in BiLAT, as well as an introduction to using the system. All participants were told in the introductory video that interpersonal aspects of the interaction are an important consideration for success. Participants then took the Culture Assimilator pretest assessment.

Next, they entered the game. They explored the "prep room" to learn about the scenario and the first character they were going to meet. Also, the goals for the meeting were introduced, including the social goal for those participants in the social goal condition. After leaving the "prep room," the Information Integration items for that character were administered. Participants then saw the meeting goals again and met with the first character until an agreement on the negotiation was reached. If the participant did not come to an agreement within forty-five minutes, the experimenter moved the participant along to the next step. At this point, participants were asked to list their goals for the meeting in free text and then took the Information Integration items for that character again as a posttest. Following this posttest, participants 
repeated this procedure with a second character who was part of the same scenario. Finally, participants took the Culture Assimilator posttest.

\section{Results}

While 59 participants completed the study, we dropped 5 participants due to computer error or complete lack of engagement. In our final analyses, we compared 25 participants in the social goal group to 29 participants playing with the standard taskrelated game objectives.

$H 1$ stated that we would observe learning gains from playing the game. To analyze the data we conducted a paired t-test with test time (pretest and posttest) as the factor. On the Information Integration items, participants were given a point for each item that matched a subject matter expert's rating of the information. Students showed significant overall learning from pre to post $(t(49)=9.213, p=.004)$. On the Culture Assimilator, participants were given a point for each item that matched the most appropriate response from the validated measure. On this measure as well there was significant overall learning from pre to post $(t(47)=4.582, p=.038)$.

$H 2$ stated that the social goals group would have more social goals for interacting with the virtual characters. To investigate, we coded participants' answers to their free-response goals for the meeting. Students in the task condition saw two task goals and reported on average 1.83 goals. Students in the social condition saw these two task goals and a third (social) goal, "Come to understand Farid's point of view," but reported significantly fewer goals on average $\left(m=1.71, \chi^{2}(1, N=54)=53.189\right.$, $p<.001)$. Interestingly, a number of participants in the task condition reported a similar or related social goal. Because the main focus of our experiment was to investigate the effects of playing the game while holding social goals, we categorized participants as "no reported social goals" and "reported social goals", regardless of their condition. Two independent coders rated anything that focused on social interaction with the virtual character in the "reported social goals" category. Table 1 has examples of reported social goals.

\begin{tabular}{|l|l|}
\hline Task goals condition & Social goal condition \\
\hline Establish a good relationship & To establish a positive personal relationship with Farid \\
\hline Build trust & Maintaining a strong personal rapport with Farid. \\
\hline $\begin{array}{l}\text { Establish a trustworthy relationship between } \\
\begin{array}{l}\text { Farid in myself. I wanted to get support and } \\
\text { unity with the Iraqi police but I also wanted } \\
\text { to attend to Farid's needs in order to keep } \\
\text { the relationship open and favorable. }\end{array}\end{array}$ & $\begin{array}{l}\text { Building the foundations for a long term relationship } \\
\text { with Farid. }\end{array}$ \\
\hline
\end{tabular}

Table 1: Reported social goals from each condition

Reporting a social goal was not significantly related to the demographic characteristics we measured: age, negotiating experience, knowledge of Arab cultures, or frequency of playing games. Neither did the reported social goal 
participants have higher pre-test scores on the Information Integration items or the Culture Assimilator (all $\mathrm{p}>.2$ ). The number of participants with reported social goals was significantly influenced by condition according to a chi square test $\left(\chi^{2}(1, N=54)\right.$ $=5.868, p=.015$ ). Table 2 shows the participants with social goals by condition.

\begin{tabular}{|l||r|r||r|}
\hline \multicolumn{1}{|c||}{$\begin{array}{l}\text { Given } \\
\text { Condition }\end{array}$} & \multicolumn{2}{c||}{ Reported goals } & \multirow{2}{*}{ Total } \\
\cline { 2 - 4 } & No social & \multicolumn{1}{l|}{ Social } & \\
\hline Task & 20 & 9 & 29 \\
\hline \hline Social & 9 & 16 & 25 \\
\hline Total & 29 & 25 & 54 \\
\hline
\end{tabular}

Table 2: Number of participants by condition and reported goals

H4 stated that students with social goals would perform better on overall measures of learning. We examined all of the learning results with respect to the learner's given goals (social vs. task). To analyze the data we conducted repeated measures analysis of variance (ANOVAs) with condition as the between-subjects factor and test time (pretest and posttest) as the within-subject factor. Here, our hypothesis was not confirmed. However, because almost a third of all participants did not report their goals as we expected based on the condition they were given, we examined all the learning results again with respect to the learner's reported goals from the manipulation check ("reported social goals" vs. "no reported social goals"). We added "reported social goals" as a between-subjects variable to repeated measures ANOVAs with test time (pretest and posttest) as the within-subject factor. On the Information Integration items, the ANOVA showed that reported social goals significantly influenced learning $(F(1,49)=3.979, p=.052)$. An ANCOVA model of the Culture Assimilator test also showed that reported social goals significantly influenced learning $(F(1,47)=8.314, p=.006)$. On both assessments, learners who reported social goals outperformed learners who did not report social goals.

H3 stated that students with social goals would have more social patterns of play in the game. To examine this hypothesis, we did an investigation into participants' actions taken in the game and compared them across reported goals. Participants who reported social goals took significantly fewer total actions in the game than participants who did not report social goals (see Table 3 for all statistics). Broken down by action type, these participants took significantly fewer business actions, while taking a statistically similar number of social actions. They also held fewer meetings before they achieved their objectives. Participants with reported social goals also took significantly fewer unique communication actions. However, although time on task was not strictly controlled by the experimenters, there were no significant differences between groups in the amount of time the game was played. 


\begin{tabular}{|l|l|l|l|l|l|l|}
\hline & $\mathbf{t}(\mathbf{5 2})$ & $\mathbf{p}$ & $\mathbf{M}_{\text {social }}$ & $\mathbf{S D}_{\text {social }}$ & $\mathbf{M}_{\text {no-social }}$ & $\mathbf{S D}_{\text {no-social }}$ \\
\hline Total actions & 2.45 & .018 & 79.0 & 27.0 & 107.0 & 49.0 \\
\hline Business actions & 2.60 & .012 & 40.0 & 16.1 & 29.6 & 12.6 \\
\hline Social actions & 1.82 & .073 & 50.1 & 21.3 & 67.1 & 42.2 \\
\hline Meetings & 2.04 & .046 & 2.8 & 1.6 & 3.9 & 2.3 \\
\hline Time played & 0.23 & .8 & 46.2 & 10.3 & 47.0 & 13.6 \\
\hline Unique actions & 2.24 & .029 & 37.0 & 7.1 & 43 & 8.6 \\
\hline
\end{tabular}

Table 3: Gameplay differences between "reported social goals" groups

\section{Discussion}

Our four hypotheses were confirmed, although not in the way that we expected. First, we found that overall, participants did learn from interacting with the virtual humans in BiLAT. Although in this study we did not compare to a more traditional classroom condition, showing that such a system can produce increases on a validated assessment is a win given the current state of evaluation in the field [11]. The results of the explicit goals manipulation in the study require closer consideration. Relevant to $H 2$, a significantly higher percentage of participants who were explicitly given a social goal in the game interface did report having a social goal as a meeting objective than those who did not see such a goal. Therefore, some students in the social goal condition did appear to be influenced to consider such goals as important. However, a third of that condition did not seem to heed the manipulation. These students may not have understood how to achieve the social goal they were given, or may not have wanted to achieve it. Additionally, unlike other similar learning orientation manipulations, simply attempting to manipulate students' social goals was not sufficient to be beneficial to learning.

What we saw instead, confirming $H 3$, was that learners with self-reported social goals for the interaction had increased learning over those who did not. The results of this study extend two sets of seemingly conflicting evidence. Okita et al. suggest that students need to believe that virtual characters are real to benefit from interacting with them $[12,16]$. However, the Media Equation [15] suggests that social responses to virtual characters are automatic. We saw that participants with self-reported social goals, regardless of condition, learned more about the scenario and characters and were better able to transfer their knowledge to novel situations. Thus, it appeared that while learners did not need to believe that the virtual humans were real humans to learn, having social goals for the interaction was beneficial.

Additionally, we saw that students who reported a social goal played the game in a qualitatively different way (confirming $H 4$ ). In an identical amount of time in the game, they took fewer actions, which may indicate that they spent more time reflecting on each action to consider their partners' perspective. They took fewer total actions relating to business and a higher percentage of social actions than students who did not report social goals. Additionally, they took fewer unique actions, signifying less exploration of the conversation space (seemingly avoiding dialog 
actions that could potentially be seen as offensive). Together, these patterns seem to present a social orientation towards gameplay, where participants hold some theory of mind about their virtual partner. This outlook is in contrast to a prominent view of learning from gameplay, which involves exploring a risk-free, task-oriented environment for discovery (as suggested by the PsychoSocial Moratorium principle and other theories of game-based learning) [7, 9]. This principle states that games are a place where learners take risks they would not normally be comfortable taking in the real world. In BiLAT, learners might manifest this principle by intentionally offending the virtual character, or experimenting with all available actions in an attempt to understand the boundaries of acceptable behavior. This type of gameplay would be at odds with a social perspective, in which learners would carefully consider their partner's perspective, attempt to avoid giving offense, and avoid exploration that would take them into unknown territory of culturally acceptable behaviors.

Our results show that a social orientation towards interaction with virtual humans in learning intercultural negotiation and perhaps similarly complex social skills may not always happen, but when it does, it leads to increased learning. The results can guide us towards designing improved ways to support social learning through virtual environments. It appears that while learning social skills in virtual environments can be more effective if we can promote a social orientation, presenting explicit social goals is not the most effective way of doing so. Instead, our search continues for ways to promote a social orientation more implicitly. A second avenue for future research is to investigate those students who arrive at a learning environment already holding social goals. Reporting a social goal was not tied to any of the demographics that we measured (e.g., prior negotiation training, knowledge of Arab cultures), nor did these participants appear to be of higher ability based on their pretest scores. It may be that other measures such as social intelligence or personality traits can provide a better characterization of these learners. We intend to build a model of how social goals are influenced by and interact with these learner characteristics. Then, we will investigate how social goals can be promoted for those students who do not already hold them.

Acknowledgments Thanks to Eric Forbell for his great effort at support and implementation. Part of this work has been sponsored by the U.S. Army Research, Development, and Engineering Command (RDECOM). Statements and opinions expressed do not necessarily reflect the position or the policy of the United States Government, and no official endorsement should be inferred. The research was supported by the Institute of Education Sciences, U.S. Department of Education, through Grant R305B040063 to CMU.

\section{References}

1. Bailenson, J., Blascovitch, J., Beall, A., Loomis, J. (2003). Interpersonal Distance in Immersive Virtual Environments. Personality and Social Psych. Bulletin, Vol. 29, No. 7, 819-833.

2. Chou, C-Y., Chan, T-W., \& Lin, C-J. (2003). Redefining the learning companion: the past, present, and future of educational agents. Computers \& Education, 40, 255-269. 
3. Cohen, Ivry, \& Keele (1992). Attention and structure in sequence learning. Journal of experimental psychology. Learning, memory, and cognition, 16 (1).

4. Cushner, K., Brislin, R, (1995). Intercultural Interactions: A Practical Guide. Second Edition. Thousands Oaks, CA: Sage Publications, Inc

5. Dweck, C.S., \& Leggett E.L. (1988). A social-cognitive approach to motivation and personality. Psychological Review, 95 256-273.

6. Gaertner, S. L., Dovidio, J. F., Anastasio, P. A., Bachman, B. A., \& Rust, M. C. (1993). The common ingroup identity model: Recategorization and the reduction of intergroup bias. European Review of social Psychology, Vol. 4, pp. 1-26.

7. Gee, J. P. (2003). What video games have to teach us about learning and literacy. New York: Palgrave/Macmillan.

8. Hill, R.W., Belanich, J., Lane, H.C., Core, M.G., Dixon, M., Forbell, E., Kim, J., \& Hart, J. (2006). Pedagogically Structured Game-based Training: Development of the ELECT BiLAT Simulation, Proc. 25th Army Science Conf.

9. Johnson, S. (2006). Everything bad is good for you: How today's popular culture is actually making us smarter. New York: Riverhead Books.

10.Landis, D., Bennett, J., \& Bennett, M. J. (2003). Handbook of Intercultural Training (3rd ed.). Sage Publications, Inc.

11.Ogan, A. \& Lane, H.C. (in press). Virtual Learning Environments for Culture and Intercultural Competence. Handbook of Intercultural Training.

12.Okita, S.Y., Bailenson, J., Schwartz, D. L., (2008). Mere Belief of Social Action Improves Complex Learning In S. Barab,K. Hay, D. Hickey (Eds.),Proc. of the 8th Int. Conf. for the Learning Sciences.

13.Prentice, D. A., \& Miller, D. T. (1999). The psychology of cultural contact. In D. A. Prentice \& D. T. Miller (Eds.), Cultural divides: Understanding and overcoming group conflict (pp. 1-19).

14.Raybourn, E. M., \& Waern, A. (2004). Social Learning Through Gaming. In Extended Abstracts of CHI Proceedings 2004. ACM Press.

15.Reeves, B., and Nass, C. (1996). The Media Equation: How People Treat Computers, Television, and New Media like Real People and Places. Cambridge University Press.

16.Rosé, C. P., \& Torrey, C. (2005). Interactivity versus Expectation: Elicting Learning Oriented Behavior with Tutorial Dialogue Systems, Proc. Intl Conf. on HCI (Interact '0.5).

17.Schunk, D. H., Pintrich, P. R., \& Meece, J. (2007). Motivation in Education: Theory, Research, and Applications (3rd ed.). Prentice Hall.

18.Swartout, W., Gratch, J., Hill, R., Hovy, E., Marsella, S., Rickel, J., \& Traum, D. (2006). Toward virtual humans. AI Magazine 27(2), 96-108.

19.Taylor, D. M., \& Moghaddam, F. M. (1994). Theories of intergroup relations: International social psychological perspectives. 2nd. Ed. New York: Praeger

20.Urdan, T., Maehr, M. (1995) Beyond a Two-Goal Theory of Motivation and Achievement: A Case for Social Goals. Review of Educational Research, Vol. 65, No. 3, 213-243.

21.Weingart, L. R., Bennett, R. J., \& Brett, J. M. (1993). The impact of consideration of issues and motivational orientation on group negotiation process and outcome. J. of Applied Psych, 78(3), 504-517. 